\title{
Compressed Sensing-Sensitivity Encoding (CS-SENSE) Accelerated Brain Imaging: Reduced Scan Time without Reduced Image Quality
}

\author{
(D).E. Vranic, (DN.M. Cross, (D). Wang, DD.S. Hippe, DE. de Weerdt, and (DM. Mossa-Basha
}

\begin{abstract}
BACKGROUND AND PURPOSE: Compressed sensing-sensitivity encoding is a promising MR imaging acceleration technique. This study compares the image quality of compressed sensing-sensitivity encoding accelerated imaging with conventional MR imaging sequences.

MATERIALS AND METHODS: Patients with known, treated, or suspected brain tumors underwent compressed sensing-sensitivity encoding accelerated 3D T1-echo-spoiled gradient echo or 3D T2-FLAIR sequences in addition to the corresponding conventional acquisition as part of their clinical brain MR imaging. Two neuroradiologists blinded to sequence and patient information independently evaluated both the accelerated and corresponding conventional acquisitions. The sequences were evaluated on 4- or 5-point Likert scales for overall image quality, SNR, extent/severity of artifacts, and gray-white junction and lesion boundary sharpness. SNR and contrast-to-noise ratio values were compared.
\end{abstract}

RESULTS: Sixty-six patients were included in the study. For T7-echo-spoiled gradient echo, image quality in all 5 metrics was slightly better for compressed sensing-sensitivity encoding than conventional images on average, though it was not statistically significant, and the lower bounds of the $95 \%$ confidence intervals indicated that compressed sensing-sensitivity encoding image quality was within $10 \%$ of conventional imaging. For T2-FLAIR, image quality of the compressed sensing-sensitivity encoding images was within $10 \%$ of the conventional images on average for 3 of 5 metrics. The compressed sensing-sensitivity encoding images had somewhat more artifacts $(P=.068)$ and less gray-white matter sharpness $(P=.36)$ than the conventional images, though neither difference was significant. There was no significant difference in the SNR and contrast-to-noise ratio. There was $25 \%$ and $35 \%$ scan-time reduction with compressed sensing-sensitivity encoding for FLAIR and echo-spoiled gradient echo sequences, respectively.

CONCLUSIONS: Compressed sensing-sensitivity encoding accelerated 3D T1-echo-spoiled gradient echo and T2-FLAIR sequences of the brain show image quality similar to that of standard acquisitions with reduced scan time. Compressed sensing-sensitivity encoding may reduce scan time without sacrificing image quality.

ABBREVIATIONS: CNR = contrast-to-noise ratio; $C S=$ compressed sensing; SENSE = sensitivity encoding; SPGR = echo-spoiled gradient echo

$\mathbf{T}$ he excellent soft-tissue contrast resolution and specialized sequences targeting different aspects of pathophysiology make MR imaging the optimal technique for studying the brain. Despite the many advantages of brain MR imaging, MR imaging acquisition is a time-consuming endeavor compared with CT. Long im-

Received May 17, 2018; accepted after revision October 22.

From the Department of Radiology (J.E.V., N.M.C., D.S.H., M.M.-B.), University of Washington, Seattle, Washington; and Philips Healthcare (Y.W., E.d.W.), Best, the Netherlands.

Please address correspondence to Mahmud Mossa-Basha, MD, University of Washington Medical Center, 1959 NE Pacific St, Seattle, WA 98195; e-mail: mmossab@uw.edu; @mossabas

Indicates article with supplemental on-line tables.

Indicates article with supplemental on-line photo.

http://dx.doi.org/10.3174/ajnr.A5905 age-acquisition times limit both the clinical application and practicality of MR imaging, particularly in medically unstable and pediatric patients.

MR imaging acquisition time is largely influenced by the number of data points sampled from $k$-space, the way these data points are sampled, and the way in which image reconstruction is performed. Several image-acquisition and postprocessing techniques have been developed to reduce image-acquisition time while still preserving image quality. ${ }^{1,2}$ These include parallel MR imaging and compressed sensing (CS) MR imaging techniques, which rely on different reconstruction constraints to accelerate image production. ${ }^{3}$ Combining these techniques can lead to image-acquisition acceleration factors that far exceed what is achievable by either parallel or CS MR imaging alone. ${ }^{3-6}$ This combined image-acceleration technique is referred to as CS-sensitivity encoding (SENSE) MR 


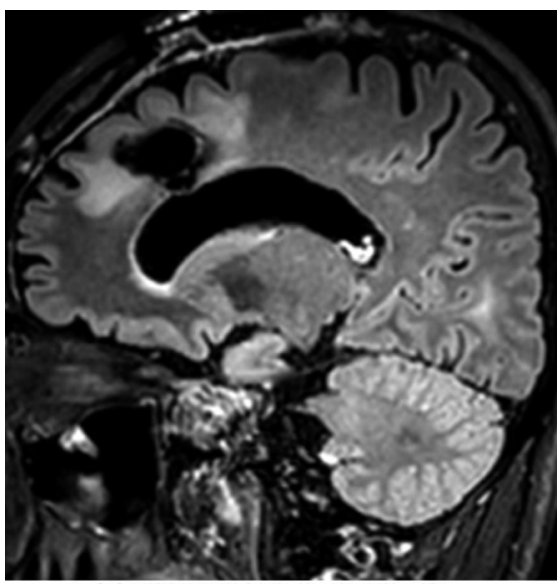

CONVENTIONAL 3D FLAIR 4:43 minutes

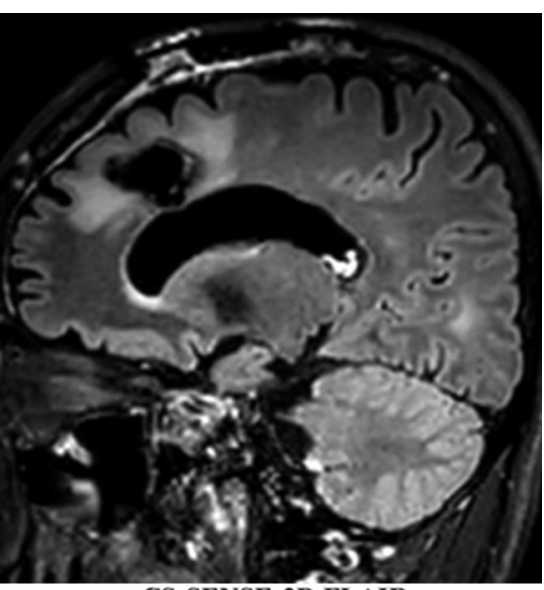

CS-SENSE 3D FLAIR

3:36 minutes

FIG 1. Conventional and CS-SENSE accelerated sagittal 3D T2-FLAIR images from the same patient demonstrate a treated primary brain tumor within the left frontal lobe. Note the sharp borders of the brain parenchymal lesion detected in both images, while CS-SENSE 3D FLAIR (right) was acquired with a $25 \%$ scan time reduction.

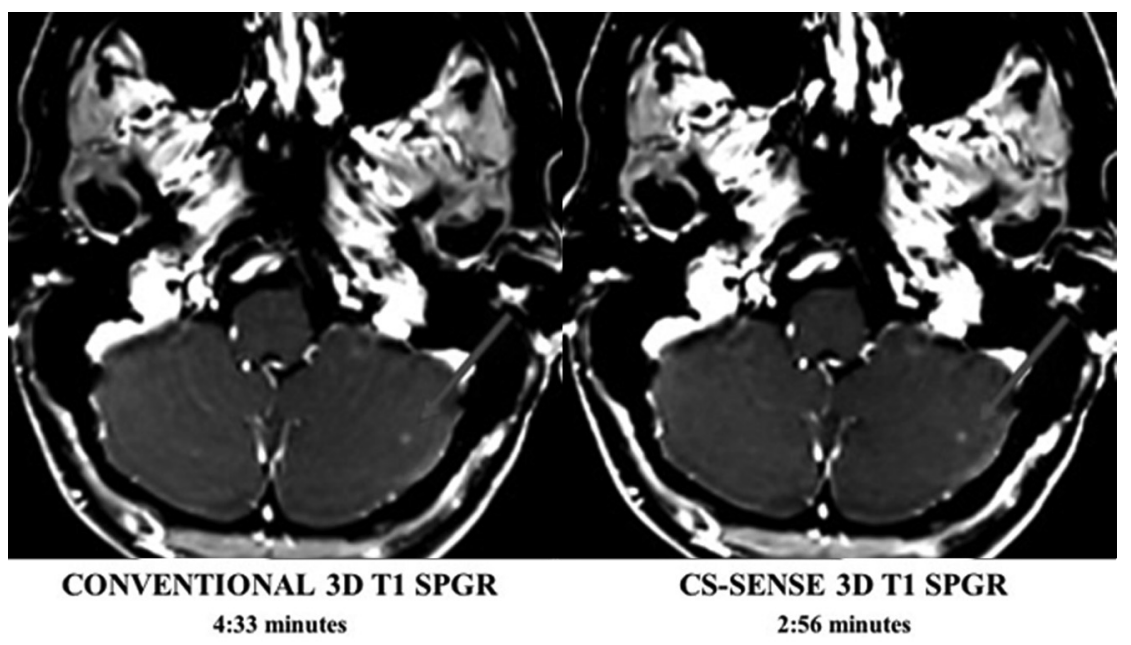

FIG 2. Conventional and CS-SENSE accelerated axial TI-SPGR images are from the same patient. The arrow demonstrates a small metastasis within the left cerebellar hemisphere that was detected by both sequences equally well. Acquisition of the CS-SENSE SPGR (right) was $35 \%$ faster than the conventional SPGR (left).

imaging, and it has the potential to dramatically decrease overall imaging times while still preserving image quality.

Despite the many technical advancements that have been made in accelerating MR imaging acquisition and image reconstruction, robust evaluation of these acceleration techniques in clinical practice is still warranted. Clinical verification of the ability of these accelerated image-acquisition techniques to produce diagnostic-quality images of the central nervous system is essential before broader implementation of these imaging techniques into clinical practice can occur. Only a small number of studies have investigated the performance of CS-SENSE MR imaging in limited patient populations as it relates to body imaging. ${ }^{4,5}$ Very few studies have evaluated CS in brain MR imaging, with the studies performed focusing on the evaluation of multiple sclerosis lesions on T2-FLAIR, ${ }^{7}$ brain MR imaging quality assessment in healthy controls, ${ }^{8}$ and evaluating achievable acceleration, reconstruction schemes, and artifacts generated from retrospective CS. ${ }^{9}$

To date, however, no one has critically evaluated the clinical performance of the integrated CS-SENSE algorithm for MR imaging applied to imaging of the central nervous system, to our knowledge. In addition, we present the first work to apply CS acceleration in a brain tumor clinical population. The purpose of the current study was to compare the image quality of CS-SENSE accelerated 3D T1echo-spoiled gradient echo (SPGR) (CS-SENSE SPGR) and T2-FLAIR (CSSENSE FLAIR) sequences with the corresponding conventional acquisitions. We hypothesized that CS-SENSE accelerated sequences will have image quality equivalent to that of conventional acquisitions while accelerating imaging.

\section{MATERIALS AND METHODS \\ Patient Selection}

With our institutional review board approval and after obtaining informed written consent, adult patients (18 years of age or older) were prospectively scanned between February 8, 2017, and January 19, 2018, for assessment of the MR imaging brain tumor protocols of our institution with inclusion of a conventional sequence and a corresponding CS-SENSE accelerated acquisition when the clinical schedule permitted. CS-SENSE accelerated acquisition was performed before or after the corresponding conventional acquisition in alternating order (to mitigate potential bias from ordering effects), with both sequences performed after gadolinium administration for both T2-FLAIR and SPGR.

\section{Image Acquisition}

All imaging was performed on a 3T Ingenia MR imaging scanner (Philips Healthcare, Best, the Netherlands) using a 16-channel head coil (In Vivo, Gainesville, Florida). Each patient underwent the brain tumor imaging protocol of our institution. This included the following sequences: axial DWI, axial T1-spin-echo, sagittal 3D T2-FLAIR with gadolinium, axial 3D T1-SPGR with gadolinium, and coronal and axial T1-spin-echo with gadolinium. In addition to these conventional acquisitions, each patient underwent either a CS-SENSE accelerated 3D T2-FLAIR (Fig 1) or a CS-SENSE accelerated gadolinium-enhanced 3D T1-SPGR sequence (Fig 2), which was performed during their routine MR imaging examination. The sequence scan parameters for both the conventional and CS-SENSE MR imaging sequences are listed in Table 1. The CS-SENSE FLAIR and CS-SENSE SPGR sequences had acceleration factors of 1.3 and 1.7 with scan time reduc- 
Table 1: Imaging parameters for CS-SENSE accelerated and conventional MRI sequences

\begin{tabular}{|c|c|c|c|c|}
\hline & 3D T2-FLAIR & CS-SENSE 3D T2-FLAIR & 3D TI-SPGR & CS-SENSE 3D TI-SPGR \\
\hline FOV $(\mathrm{cm})$ & $230 \times 2230 \times 150$ & $230 \times 230 \times 150$ & $240 \times 240 \times 168$ & $240 \times 240 \times 168$ \\
\hline True voxel size $\left(\mathrm{mm}^{3}\right)$ & $1 \times 1 \times 2$ & $1 \times 1 \times 2$ & $1.1 \times 1.1 \times 1.1$ & $1.1 \times 1.1 \times 1.1$ \\
\hline Interpolated voxel $\left(\mathrm{mm}^{3}\right)$ & $1 \times 1 \times 1$ & $1 \times 1 \times 1$ & $1 \times 1 \times 0.5$ & $1 \times 1 \times 0.5$ \\
\hline TR/TE (ms) & $25 / 3.6$ & $25 / 3.6$ & $4800 / 301$ & $4800 / 301$ \\
\hline Slices (No.) & 150 & 150 & 300 & 300 \\
\hline $\mathrm{TI}(\mathrm{ms})$ & - & - & 1650 & 1650 \\
\hline SENSE acceleration factor (RL, FH) & $(2,1)$ & $(2,1)$ & $(2.4,1.7)$ & $(2.4,1.7)$ \\
\hline CS acceleration factor & - & 1.3 & - & 1.7 \\
\hline Total acceleration factor & 2 & 3.4 & 4.1 & 5.3 \\
\hline Scan time (min:sec) & $4: 33$ & $3: 36$ & $4: 43$ & $2: 56$ \\
\hline Scan time reduction & - & $25 \%$ & & $35 \%$ \\
\hline
\end{tabular}

Note:-RL indicates Right-to-left; FH, Foot-to-head; -, Not used/no value.

tions of $25 \%$ and $35 \%$ compared with the conventional acquisition counterparts, respectively. These CS-SENSE accelerated acquisitions used a balanced variable density incoherent undersampling acquisition scheme and iterative reconstruction to solve an inverse problem with a sparsity constraint. Specifically, the images were acquired using a random undersampling pattern with the Poisson disc style distribution. Image reconstruction was performed using a wavelet transform for the sparsity term, according to the common CS and parallel imaging problem definitions. Prior knowledge of noise decorrelation, regularization, and coil sensitivities was used to provide an optimal SNR as a starting point, allowing additional acceleration capabilities via sparsity constraining. The reconstruction algorithm was based on a modified fast iterative shrinkage/ soft thresholding algorithm (FISTA) scheme, ${ }^{10}$ which entails iterative reconstruction. Conventional clinical acquisition T2-FLAIR and T1-SPGR sequences served as imaging control sequences against which the CS-SENSE FLAIR and SPGR sequences could be compared.

\section{Image Evaluation}

Two experienced board-certified neuroradiologists (M.M.-B. and N.M.C.) blinded to the imaging technique and patient clinical information independently evaluated all CS-SENSE and corresponding conventional sequences. All imaging studies were deidentified and randomized so that each rater was unaware of whether they were reviewing a CS-SENSE or conventional acquisition. Raters evaluated overall imaging quality on the following 4-point scale: 1, nondiagnostic; 2, limited but interpretable; 3 , minimally limited; and 4, optimal quality. Image SNR was rated on the following 5-point scale: 1, markedly diminished SNR that renders the images uninterpretable; 2 , moderately diminished SNR that affects interpretation; 3, diminished SNR that only mildly limits interpretation; 4, mildly diminished SNR that does not affect image interpretation; and 5, optimal SNR. Image artifacts were evaluated on the following 5-point scale: 1 , severe image artifacts; 2 , moderate artifacts; 3 , mild artifacts; 4 , trace artifacts; and 5, no artifacts. Brain parenchymal lesion border sharpness was evaluated on the following 3-point scale: 1 , a lesion whose borders were indistinguishable from background brain; 2, a lesion with blurry margins; and 3, sharp lesion margins. Finally, gray-white matter sharpness was assessed using the following 3-point scale: 1, indistinguishable gray-white sharpness; 2 , blurry gray-white sharpness; and 3, well-defined gray-white sharpness.
In addition to qualitative image evaluation, quantitative evaluation of SNR and the contrast-to-noise ratio (CNR) was also performed for each CS-SENSE and conventional acquisition, respectively. For each patient, ROIs were drawn on representative images from each CS-SENSE and conventional acquisition. ROIs were positioned within the normal subcortical white matter, within a focal brain lesion, and outside the patient, in what was classified as image background. All ROIs were the same size and had nearly identical positioning between sequences. As reported elsewhere in the literature, ${ }^{7,11}$ $\mathrm{SNR}$ and CNR were calculated as follows: $\mathrm{SNR}=\mathrm{SI} / \mathrm{SD}_{\text {noise }}$ and $\mathrm{CNR}=\left(\mathrm{SI}_{\text {lesion }}-\mathrm{SI}_{\mathrm{WM}}\right) / \mathrm{SD}_{\text {noise }}$, where $\mathrm{SI}$ is the average signal intensity of the lesion or white matter and $\mathrm{SD}_{\text {noise }}$ is the $\mathrm{SD}$ of noise.

\section{Statistical Analysis}

While raters evaluated images using 4- or 5-point scales, they ultimately only used 2-3 levels of each scale, with the middle level being the most common. To improve interpretability, we dichotomized all scales, mainly to get the best possible balance of ratings above and below the threshold. Specifically, image quality was dichotomized as optimal image quality (5 versus $1-4$ ), optimal SNR (5 versus $1-4)$, no or trace artifacts ( $4-5$ versus $1-3$ ), sharp gray-white matter boundaries (3 versus $1-2$ ), and sharp lesion boundaries (3 versus 1-2).

The percentages for each image-quality metric were compared between CS-SENSE and the corresponding conventional images (FLAIR versus CS-FLAIR, SPGR versus CS-SPGR) using the nonparametric bootstrap to calculate $95 \% \mathrm{CI}$ and $P$ values for the differences. The widths of the $95 \%$ CIs were used to help assess a plausible range of differences in image quality between CS-SENSE and the corresponding conventional images. Ratings from both raters were analyzed together for the primary analysis and separately as a sensitivity analysis. Bootstrap resampling was performed by patient to account for the nonindependence of ratings by both raters of the same images and for multiple scans acquired from some patients.

Interrater agreement was assessed using the Cohen $\kappa$ and by counting how often both raters, 1 rater, and neither rater rated CS-SENSE images at least as highly as conventional images. All statistical calculations were conducted with R statistical and computing software (Version 3.1.1; http://www.r-project.org/). Throughout, 2-tailed tests were used with statistical significance defined as $P<.05$. 
Table 2: CS-SENSE and conventional MRI sequence qualitative evaluation

\begin{tabular}{|c|c|c|c|c|c|c|c|c|c|c|}
\hline \multirow[b]{3}{*}{ Metric } & \multicolumn{5}{|c|}{ SAG FLAIR ( $n=70$ Reads) } & \multicolumn{5}{|c|}{$\operatorname{SPGR}(n=68$ Reads) } \\
\hline & \multicolumn{2}{|c|}{ Acquisition $^{\mathrm{a}}$} & \multicolumn{3}{|c|}{ Difference } & \multicolumn{2}{|c|}{ Acquisition $^{\mathrm{a}}$} & \multicolumn{3}{|c|}{ Difference } \\
\hline & CS-SENSE & Conventional & Mean & $(95 \% \mathrm{Cl})$ & $P$ Value & CS-SENSE & Conventional & Mean & $(95 \% \mathrm{Cl})$ & $P$ Value \\
\hline Optimal image quality & $19(27.1)$ & $19(27.1)$ & $0.0 \%$ & $(-510.0-10.0)$ & $>.99$ & $34(50.0)$ & $25(36.8)$ & $13.2 \%$ & $(-5.7-31.8)$ & .15 \\
\hline Optimal SNR & $6(8.6)$ & $5(7.1)$ & $1.4 \%$ & $(-55.7-8.6)$ & .67 & $27(39.7)$ & $24(35.3)$ & $4.4 \%$ & $(-59.7-18.2)$ & .55 \\
\hline No or trace artifacts & $52(74.3)$ & $60(85.7)$ & $-511.4 \%$ & $(-524.3-0.0)$ & .068 & $46(67.6)$ & $37(54.4)$ & $13.2 \%$ & $(-53.0-28.6)$ & .11 \\
\hline Sharp gray-white matter boundaries & $36(51.4)$ & $39(55.7)$ & $-54.3 \%$ & $(-512.9-4.3)$ & .36 & $27(39.7)$ & $26(38.2)$ & $2.2 \%$ & $(-58.1-12.2)$ & .67 \\
\hline Sharp lesion boundaries & $57(86.4)$ & $55(83.3)$ & $2.3 \%$ & $(-57.1-11.7)$ & .65 & $52(83.9)$ & $52(83.9)$ & $0.8 \%$ & $(-58.6-10.0)$ & .84 \\
\hline
\end{tabular}

Note:-SAG indicates sagittal.

a Values are No. (\%) where higher percentages indicate a higher prevalence of good image quality.

Table 3: CS-SENSE and conventional MRI interrater agreement

\begin{tabular}{|c|c|c|c|c|c|c|c|c|}
\hline \multirow[b]{3}{*}{ Metric } & \multicolumn{4}{|c|}{ SAG FLAIR ( $n=35$ Exams) } & \multicolumn{4}{|c|}{ AX SPGR ( $n=34$ Exams) } \\
\hline & \multicolumn{2}{|c|}{ CS-SENSE } & \multicolumn{2}{|c|}{ Conventional } & \multicolumn{2}{|c|}{ CS-SENSE } & \multicolumn{2}{|c|}{ Conventional } \\
\hline & $\kappa$ & $(95 \% \mathrm{Cl})$ & $\kappa$ & $(95 \% \mathrm{Cl})$ & $\kappa$ & $(95 \% \mathrm{Cl})$ & $\kappa$ & $(95 \% \mathrm{Cl})$ \\
\hline Optimal image quality & 0.20 & $(0.00-0.43)$ & 0.17 & $(-0.07-0.43)$ & 0.32 & $(0.00-0.59)$ & 0.57 & $(0.26-0.83)$ \\
\hline Optimal SNR & 0.00 & a & -0.07 & a & 0.12 & $(-0.19-0.42)$ & 0.11 & $(-0.18-0.42)$ \\
\hline Trace or no artifacts & 0.41 & $(0.07-0.72)$ & -0.14 & a & 0.60 & $(0.26-0.86)$ & 0.50 & $(0.28-0.74)$ \\
\hline Sharp gray-white matter boundaries & 0.01 & a & 0.02 & $(-0.14-0.16)$ & -0.10 & $(-0.30-0.04)$ & -0.04 & $(-0.19-0.07)$ \\
\hline Sharp lesion boundaries & 0.18 & $(0.00-0.53)$ & 0.00 & a & 0.31 & $(-0.09-0.69)$ & 0.33 & $(0.00-0.70)$ \\
\hline
\end{tabular}

Note:-AX indicates axial; SAG, sagittal.

${ }^{a}$ The $95 \%$ confidence interval was not provided when ratings were too uncommon to calculate reliably with the bootstrap.

\section{RESULTS}

\section{Patient Data}

Sixty-nine patients were reviewed. Three patients were scanned with 1 of the 2 accelerated image-acquisition sequences but were not scanned with the corresponding conventional sequence and were excluded from analysis. This step resulted in a final cohort of 66 patients. Of these 66 patients, 35 patients were imaged with CS-SENSE FLAIR for a total of 35 scans (16 women [45.7\%]; 25-74 years of age; median, 47 years). Thirty-one patients were imaged with the CS-SENSE SPGR, with 1 patient being scanned twice and a second patient being scanned 3 times, each at different time points, resulting in a total of 34 CS-SENSE SPGR scans (16 women [51.6\%]; $18-83$ years of age; median, 63 years). Ninety-five percent of patients included in this cohort demonstrated brain lesions on MR imaging (On-line Table 1). There were a total of 89 and 56 lesions on FLAIR and SPGR, respectively. There were lesions in 34 patients (34 scans) in the FLAIR cohort, 22 of whom had multiple lesions. Lesions were also present in 29 patients (32 scans) in the SPGR cohort, 10 of whom had multiple lesions. Lesion sizes are summarized in On-line Table 2.

\section{Qualitative Image Comparison}

Pooled image-quality ratings are summarized in Table 2. For FLAIR, there were no statistically significant differences in overall image quality, SNR, gray-white matter boundary sharpness, or lesion-border sharpness between CS-SENSE and conventional sequences, with the lower bound of the $95 \%$ CIs indicating that image quality of the CS-SENSE images was within approximately $10 \%$ of the conventional images by these metrics. However, there was a trend toward more artifacts on CS-SENSE compared with conventional images $(11.4 \%, P=.068)$.

For SPGR, there were no significant differences in any imagequality metric between CS-SENSE and conventional SPGR, though CS-SENSE images had slightly higher image-quality ratings on average than the conventional images. By each metric, image-quality ratings of CS-SENSE were within $10 \%$ of the conventional SPGR ratings based on the lower bound of the $95 \%$ CI. Differences in image quality were most noticeable between the CS-SENSE SPGR and the standard SPGR; 50\% of accelerated SPGR studies demonstrated optimal image quality compared with $37 \%$ of the standard SPGR acquisitions.

The image-quality results were generally similar when examined by each rater separately with a few notable exceptions. For FLAIR, rater 1 tended to rate gray-white boundary sharpness of CS-SENSE images more highly than on conventional images $(+8.6 \%, P=.14)$, while rater 2 rated CS-SENSE images lower than on conventional images $(-17.1 \%, P<.001)$. For SPGR, rater 1 rated SNR and lack of artifacts in CS-SENSE images more highly than on conventional images $(+20.6 \%, P=.026$ and $+26.5 \%, P=.027$, respectively), while rater 2 rated the images more similarly $(-11.8 \%, P=.27$ and $+0.0 \%, P>.99$, respectively).

\section{Interrater Agreement}

Interrater agreement scores for the CS-SENSE and conventional sequences are listed in Table 3. Interrater agreement for each image quality was mostly poor to fair for FLAIR $(\kappa<0.4)$ but fair to moderate for SPGR $(\kappa=0.2-0.6)$. Despite some differences in absolute ratings, raters both agreed $77 \%-91 \%$ of the time that the image-quality metrics of the CS-SENSE FLAIR were at least as good as the those of conventional images and rarely agreed that the CS-SENSE FLAIR images were worse than conventional images (Table 4 ). Similarly, raters both agreed $67 \%-87 \%$ of the time that the image-quality metrics of the CS-SENSE SPGR were at least as good as those of the conventional images, while only agreeing $0 \%-6 \%$ of the time that the conventional images were better. In terms of disagreement, there was $>1$ disagreement on the Likert scale only for artifact severity (On-line Figure), which occurred in 7/69 comparisons. This level of disagreement did not occur for any other qualitative metrics. 
Table 4: Interrater agreement on image-quality comparisons of CS-SENSE and conventional MRI ${ }^{\mathrm{a}}$

\begin{tabular}{|c|c|c|c|c|c|c|}
\hline \multirow[b]{3}{*}{ Metric } & \multicolumn{3}{|c|}{ SAG FLAIR ( $n=35$ Exams) } & \multicolumn{3}{|c|}{ AX SPGR ( $n=34$ Exams) } \\
\hline & \multicolumn{3}{|c|}{$\begin{array}{c}\text { Image Quality of } \\
\text { CS-SENSE } \geq \text { Conventional }\end{array}$} & \multicolumn{3}{|c|}{$\begin{array}{c}\text { Image Quality of } \\
\text { CS-SENSE } \geq \text { Conventional }\end{array}$} \\
\hline & Both Raters & 1 Rater & Neither Rater & Both Raters & 1 Rater & Neither Rater \\
\hline Optimal image quality & $28(80.0)$ & $7(20.0)$ & $0(0.0)$ & $26(76.5)$ & $6(17.6)$ & $2(5.9)$ \\
\hline Optimal SNR & $32(91.4)$ & $3(8.6)$ & $0(0.0)$ & $23(67.6)$ & $10(29.4)$ & $1(2.9)$ \\
\hline Trace or no artifacts & $27(77.1)$ & $5(14.3)$ & $3(8.6)$ & $29(85.3)$ & $3(8.8)$ & $2(5.9)$ \\
\hline Sharp gray-white matter boundaries & $28(80.0)$ & $7(20.0)$ & $0(0.0)$ & $26(76.5)$ & $8(23.5)$ & $0(0.0)$ \\
\hline Sharp lesion boundaries & $29(87.9)$ & $4(12.1)$ & $0(0.0)$ & $27(87.1)$ & $3(9.7)$ & $1(3.2)$ \\
\hline
\end{tabular}

Note:-SAG indicates sagittal; AX, axial.

${ }^{a}$ Values are No. (\%).

Table 5: White matter SNR and lesion-white matter CNR

\begin{tabular}{|c|c|c|c|c|c|c|c|c|c|c|}
\hline \multirow[b]{3}{*}{ Variable } & \multicolumn{5}{|c|}{ SAG FLAIR ( $n=35$ Exams) } & \multicolumn{5}{|c|}{ AX SPGR ( $n=34$ Exams) } \\
\hline & \multicolumn{2}{|c|}{ Acquisition $^{\mathrm{a}}$} & \multicolumn{3}{|c|}{ Difference } & \multicolumn{2}{|c|}{ Acquisition $^{a}$} & \multicolumn{3}{|c|}{ Difference } \\
\hline & CS-SENSE & Conventional & Value $^{\text {b }}$ & $(95 \% \mathrm{Cl})$ & $P$ Value & CS-SENSE & Conventional & Value $^{\text {b }}$ & $(95 \% \mathrm{CI})$ & $P$ Value \\
\hline White matter SNR & $68(62-89)$ & $59(51-75)$ & 8.6 & $(-0.2-17.8)$ & .062 & $43(34-69)$ & $43(28-69)$ & 0.3 & $(-10.9-13.7)$ & .75 \\
\hline Lesion-white matter CNR & $68(49-81)$ & $60(44-78)$ & 8.6 & $(-2.4-17.4)$ & .083 & $15(9-32)$ & $12(6-24)$ & 3.3 & $(-3.2-8.8)$ & .31 \\
\hline
\end{tabular}

Note:-SAG indicates sagittal; AX, axial.

${ }^{a}$ Values are median (interquartile range).

${ }^{b}$ Values are the difference in medians of the two acquisitions.

\section{Quantitative Assessment}

The white matter SNR or lesion CNR measurements were high for both conventional and CS-SENSE FLAIR acquisitions ( $\geq 44$ in all cases), though there was a trend toward higher SNR and CNR values on average for the CS-SENSE acquisition (Table 5). White matter SNR measurements were also relatively high for conventional and CS-SENSE SPGR acquisitions ( $\geq 28$ in all cases) with little numeric difference between them on average (difference in medians, $0.3 ; P=.75$ ). The lesion CNR measurements from the SPGR acquisitions tended to be lower and ranged from 9 to 24 overall. The CNR was slightly higher on average in CS-SENSE than on the conventional acquisitions (difference in medians, 3.3; $P=.31$ ), but the difference was not statistically significant (Table 5).

\section{DISCUSSION}

Long MR imaging acquisition times represent a significant limitation to widespread use of MR imaging. This is especially true for MR imaging in the evaluation of both clinically unstable and pediatric patients: Increased MR imaging scan time may expose these individuals to an increased need for sedation or result in limited diagnostic quality due to motion. Long image-acquisition times also negatively impact radiology workflow, leading to scheduling bottlenecks. Finally, long acquisition times contribute to the high cost of MR imaging. Given the significance of imageacquisition time for patient safety, clinical efficiency, image quality, and cost, technical effort has been made to decrease imageacquisition and reconstruction times. CS techniques show promise in providing imaging acceleration without significant image-quality degradation. Despite the promise these acceleration techniques hold for improving patient throughput and decreasing imaging cost, rigorous evaluation of the performance of these acceleration techniques in a clinical imaging population has yet to be undertaken. To our knowledge, this is the first study to translate CS-SENSE, which combines and integrates CS and SENSE parallel imaging, to a clinical brain tumor patient popula- tion to evaluate image quality relative to corresponding conventional MR imaging sequences.

In the current study, we hypothesized that CS-SENSE accelerated sequences would have image quality equivalent to that of standard acquisitions while accelerating imaging. To evaluate this hypothesis, we compared the clinical performance of 2 CS-SENSE accelerated MR imaging sequences with their corresponding conventional sequences in a clinical cohort undergoing brain tumor MR imaging scans. On the basis of blinded multirater evaluations of multiple clinically pertinent imaging variables, these accelerated acquisitions largely performed as well as their conventional counterparts across several image-quality metrics, including overall image quality, SNR, image artifacts, gray-white matter boundary sharpness, and parenchymal lesion border sharpness. In particular, the lower bounds of $95 \%$ CIs of the differences in image quality between CS-SENSE and conventional images indicated that the CS-SENSE images were within 10\% of the conventional images for all metrics for the SPGR sequence and for 3 of 5 metrics for the FLAIR sequence. The CS-SENSE acquisitions had no significant differences in white matter SNR and lesion CNR relative to their corresponding conventional acquisitions, and in fact, there was a trend toward higher values for the CS-SENSE FLAIR relative to the conventional FLAIR. CS-SENSE showed at least comparable SNR and CNR measures relative to their conventional counterparts. While this finding is somewhat counterintuitive considering the undersampling algorithm used by CSSENSE, increased/similar SNR is thought be a result of the denoising algorithm incorporated into CS-SENSE. CS-SENSE FLAIR and SPGR sequences decreased imaging time by $25 \%$ and $35 \%$ relative to conventional sequences, respectively. These results confirm that CS-SENSE sequences produce diagnostic-quality MR images of the brain specifically for brain tumor protocols while reducing overall image-acquisition time compared with conventional acquisitions.

To date, multiple MR imaging techniques have been developed with the goal of accelerating image-acquisition and recon- 
struction times. Examples of these acceleration techniques include parallel imaging and CS. Because parallel imaging and CS rely on different pieces of ancillary information for image production, it is possible to combine and integrate these acceleration techniques, ${ }^{1-3,5,6,12}$ with the resultant combined technique referred to as CS-SENSE MR imaging. Most interesting, integration of these 2 individual acceleration techniques is synergistic and results in an imaging-acceleration factor that exceeds the accelerations achieved by either parallel imaging or CS alone while still keeping image noise low. ${ }^{3,5}$ Liang et $\mathrm{al}^{3}$ illustrated this principle using MR imaging phantoms, demonstrating superior performance of CS-SENSE relative to parallel imaging or CS alone at increasing acceleration factors. Otazo et $\mathrm{al}^{5}$ independently came to similar conclusions when they demonstrated that a combined CS-SENSE technique resulted in a 2-fold increase in acceleration over CS alone.

Despite the increases in imaging acceleration observed with in vitro phantom imaging, little has been done to evaluate the performance of CS-SENSE acceleration techniques in a clinical patient population. Chandarana et $\mathrm{al}^{4}$ used a novel CS-SENSE accelerated T1-weighted gradient-echo technique with a goldenangle radial $k$-space sampling scheme to evaluate 8 healthy subjects undergoing multiphase liver MR imaging. The authors concluded that their CS-SENSE accelerated sequence produced diagnostic-quality images. Kayvanrad et $\mathrm{al}^{8}$ evaluated accelerated brain MR imaging using CS, parallel imaging, or low-resolution scans in 5 healthy volunteers and found that CS significantly outperformed parallel imaging and low-resolution imaging with a number of sequences. Sharma et $\mathrm{al}^{9}$ performed retrospective CS of fully acquired datasets in 15 neuroimaging datasets to determine appropriate reconstructions and delineate expected artifacts in these reconstructions; they found 2-fold acceleration to be appropriate with ringing and blurring artifacts the most commonly encountered artifacts. These studies evaluated small cohorts of healthy volunteers and not patient populations. Toledano-Massiah et $\mathrm{al}^{7}$ evaluated the clinical performance of CS 3D FLAIR in a multiple sclerosis population and demonstrated that CS produces diagnostic-quality images of the brain while reducing imaging time. In contrast to this study, which evaluated CSaccelerated FLAIR image quality, we evaluated the clinical performance of CS-SENSE acceleration of both T1- and T2-weighted sequences in a brain tumor patient population that has not previously been evaluated with these acceleration techniques.

CS-SENSE has its own inherent technical limitations. While undersampling of $k$-space is essential to decreasing image-acquisition time, if too few data points are acquired, then image quality is adversely affected. This issue manifests as decreased SNR and increased image blurring. ${ }^{3}$ Technical challenges also arise during image reconstruction. To accurately reconstruct CS-SENSE images, solution of an optimization problem with multiple mathematical constraints is required. This process proves computationally complex and time-consuming. Fortunately, immediate sensitivity coil compression to a smaller number of virtual coils can aid in reducing the time required to complete the iterative reconstruction process. ${ }^{13}$ The iteration used in the current examination reconstructs inline in $<1$ minute.

Our study had several limitations and challenges. The clinical cohort evaluated was relatively limited in size. Additionally, the current study evaluated imaging acceleration using CS-SENSE in patients undergoing an MR imaging brain tumor protocol. Individuals with other central nervous system pathologies were not included in our clinical cohort, limiting generalizability to other brain MR imaging applications. Because imaging focused on the brain, these results may not be generalizable to other pathologies or anatomic sites including spine, head and neck, and body MR imaging. Further investigation is necessary to establish the value of CS-SENSE in these anatomic regions. Interrater agreement $(\kappa)$ ranged from 0 to 0.6 for the current study across all evaluations. The raters reviewed the accelerated sequences randomly and independent of the corresponding conventional acquisition using qualitative scales, both of which likely contributed to the limited agreement. Although raters had disagreements on the actual rating, they usually agreed $>80 \%$ of the time that CS-SENSE imagequality metrics were at least as good as those for conventional images. Last, raters did not perform a diagnostic or clinical task such as detecting or characterizing lesions or changes in lesions, so further study is needed to confirm that these tasks are not adversely affected by CS-SENSE acceleration.

\section{CONCLUSIONS}

Accelerated CS-SENSE MR imaging provides equivalent image quality compared with corresponding conventional MR imaging in patients undergoing brain MR imaging of tumor by $3 \mathrm{D}$ T2FLAIR and T1-SPGR sequences based on qualitative and quantitative assessment. CS-SENSE acceleration does not appear to adversely impact overall image quality relative to the corresponding conventional acquisitions. The results of this study are consistent with our hypothesis that there are no appreciable differences between the CS-SENSE acquisitions and their conventional counterparts. On the basis of these results, use of CS-SENSE accelerated sequences in clinical practice can be considered to reduce scan time and improve throughput without loss of image quality, specifically for 3D T2-FLAIR and T1-SPGR sequences in brain tumor MR imaging protocols.

Disclosures: Daniel S. Hippe-UNRELATED: Grants/Grants Pending: National Institutes of Health (R01NS092207), GE Healthcare, Philips Healthcare, Toshiba America Medical Systems, Siemens, Comments: for statistical work on other studies.* Elwin de Weerdt-UNRELATED: Employment: Royal Philips. *Money paid to the institution.

\section{REFERENCES}

1. Chun IY, Adcock B, Talavage TM. Efficient compressed sensing SENSE pMRI reconstruction with joint sparsity promotion. IEEE Trans Med Imaging 2016;35:354-68 CrossRef Medline

2. Tsao J, Kozerke S. MRI temporal acceleration techniques. J Magn Reson Imaging 2012;36:543-60 CrossRef Medline

3. Liang D, Liu B, Wang J, et al. Accelerating SENSE using compressed sensing. Magn Reson Med 2009;62:1574-84 CrossRef Medline

4. Chandarana H, Feng L, Block TK, et al. Free-breathing contrastenhanced multiphase MRI of the liver using a combination of compressed sensing, parallel imaging, and golden-angle radial sampling. Invest Radiol 2013;48:10-16 CrossRef Medline

5. Otazo R, Kim D, Axel L, et al. Combination of compressed sensing and parallel imaging for highly accelerated first-pass cardiac perfusion MRI. Magn Reson Med 2010;64:767-76 CrossRef Medline

6. Tam LK, Galiana G, Stockmann JP, et al. Pseudo-random center placement $\mathrm{O}$-space imaging for improved incoherence compressed sensing parallel MRI. Magn Reson Med 2015;73:2212-24 CrossRef Medline 
7. Toledano-Massiah S, Sayadi A, de Boer R, et al. Accuracy of the compressed sensing accelerated 3D-FLAIR sequence for the detection of MS plaques at 3T. AJNR Am J Neuroradiol 2018 Jan 18. [Epub ahead of print] CrossRef Medline

8. Kayvanrad M, Lin A, Joshi R, et al. Diagnostic quality assessment of compressed sensing accelerated magnetic resonance neuroimaging. J Magn Reson Imaging 2016;44:433-44 CrossRef Medline

9. Sharma SD, Fong CL, Tzung BS, et al. Clinical image quality assessment of accelerated magnetic resonance neuroimaging using compressed sensing. Invest Radiol 2013;48:638-45 CrossRef Medline
10. Zibulevsky M, Elad M. L1-L2 optimization in signal and image processing. IEEE Signal Processing Magazine 2010;27:76-88 CrossRef

11. Kaufman L, Kramer DM, Crooks LE, et al. Measuring signal-tonoise ratios in MR imaging. Radiology 1989;173:265-67 CrossRef Medline

12. Liu F, Duan Y, Peterson BS, et al. Compressed sensing MRI combined with SENSE in partial k-space. Phys Med Biol 2012;57:N391403 CrossRef Medline

13. Hollingsworth KG. Reducing acquisition time in clinical MRI by data undersampling and compressed sensing reconstruction. Phys Med Biol 2015;60:R297-322 CrossRef Medline 